EESTI NSV TEADUSTE AKADEEMIA TOIMETISED. X KOIDE

FOOSIKALIS-MATEMAATILISTE JA TEHNILISTE TEADUSTE SEERIA. 1961, NR. 1

ИЗВЕСТИЯ АКАДЕМИИ НАУК ЭСТОНСКОН ССР. ТОМ Х

СЕРИЯ ФИЗИКО-МАТЕМАТИЧЕСКИХ И ТЕХННЧЕСКИХ НАУК. 1961, № 1

\title{
К ОПРЕДЕЛЕНИЮ КРИТИЧЕСКОЙ НАГРУЗКИ КОНИЧЕСКОЙ ОБОЛОЧКИ ВРАЩЕНИЯ
}

\section{П. МЮРСЕПП}

В работе при помощи метода возмущения выводится простая расчетная формула для определения критической нагрузки кругового усеченного конуса средней длины при поперечном внешнем давлении, линейно распределенном вдоль образующей конуса.

В виде частного случая получена формула для критической нагрузки равномерного поперечного давления.

Решение задачи сводим к решению уравнения (2.1) статьи [1], которое представляем в виде

$$
w^{\prime \prime \prime \prime}+\frac{6 \sin \varphi}{\varrho} w^{\prime \prime \prime}+\frac{6 \sin ^{2} \varphi}{\varrho^{2}} w^{\prime \prime}-\left(\frac{q s^{6}}{\varrho^{3} \cos ^{3} \varphi}-\frac{\lambda^{2} s^{8}}{\varrho^{6} \cos ^{2} \varphi}\right) w=0
$$

где $q=\frac{p}{E h}, w-$ прогиб, $\varphi$ - угол конусности, $Q$ - расстояние точки оболочки от оси, $p$ - интенсивность поперечного давления, $s-$ число волн по окружности, $E-$ модуль Юнга, $\lambda^{2}=\frac{h^{2}}{12\left(1-v^{2}\right)}$, причем $v-$ модуль Пуассона, $h$ - толщина оболочки.

Давление, линейно распределенное вдоль образующей конуса, можем выразить следующим образом (фиг. 1):

$$
q=q_{1} \frac{\varrho_{2}-\varrho}{\varrho_{2}-\varrho_{1}}+q_{2}=q_{1}\left(\frac{\varrho_{2}}{\varrho_{2}-\varrho_{1}}+q_{2}^{1}-\frac{1}{\varrho_{2}-\varrho_{1}} \varrho\right)=q_{1}\left(c-d_{\varrho}\right)
$$

Обозначим $\alpha=\frac{q_{1} s^{2}}{\cos ^{3} \varphi}, \beta^{2}=\frac{\lambda^{2} s^{4}}{\cos ^{2} \varphi}, a=\frac{l}{2} \sin \varphi$, где $l$ - относительная длина оболочки. Введем пере-

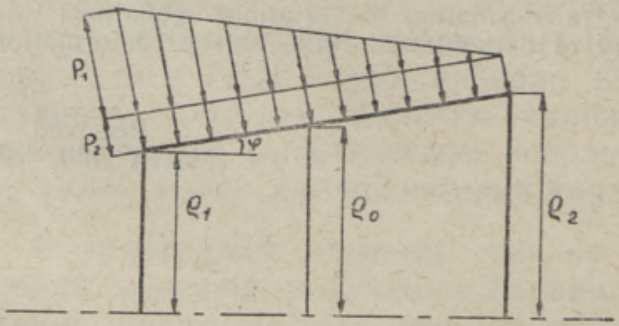

Фнг. 1 менную $\xi_{0}=2 \frac{\xi-l^{\prime}}{l}$, где $l^{\prime}-$ расстояние, измеренное вдоль образующей конуса от меньшего днища до сечения, радиус которого $\mathrm{Q}_{0}=1$ является средним геометрическим радиусов $\varrho_{1}$ и Q2. Будем считать координату $\xi_{0}$ влево от выбранного сечения отрицательной, вправо - положительной. 
Приведенные расстояния днищ конуса от сечения @o $=1$ суть $\xi^{\prime}=-2 \frac{l^{\prime}}{l}, \quad \xi^{\prime \prime}=2 \frac{l^{\prime \prime}}{l}, \quad$ их разность $\xi^{\prime \prime}-\xi^{\prime}=2$. Кроме того, $\left(1+a \xi^{\prime}\right)\left(1+a \xi^{\prime \prime}\right)=1$. Из этих условий получим

$$
\xi^{\prime}=-\left(1-\frac{a}{2}\right), \xi^{\prime \prime}=1+\frac{a}{2}, l^{\prime}=\frac{l}{2}\left(1-\frac{a}{2}\right), l^{\prime \prime}=\frac{l}{2}\left(1+\frac{a}{2}\right) .
$$

Учитывая, что $a$ - малый параметр, берем из разложений только члены до $a^{2}$ включительно. Замене переменных $\xi_{0}=2 \frac{\xi-l^{\prime}}{l}$ соответствует соотношение между дифференциальными операторами $D=\frac{2}{l} D_{0}$, где $D=\frac{d}{d \xi}, D_{0}=\frac{d}{d \xi_{0}}$.

Уравнение (1) принимает вид

$$
\left.\left\{D_{0}^{4}+\frac{6 a}{\varrho} D_{0}^{3}+\frac{6 a^{2}}{\varrho^{2}} D_{0}^{2}-\frac{l_{1}^{4}}{\varrho^{3}} \mid \alpha(c-d \varrho)-\frac{\beta^{2}}{\varrho^{3}}\right]\right\} w=0 .
$$

Здесь $l_{1}=\frac{l s}{2}$. Теперь произведем замену переменных $x=\frac{1}{a} \ln \varrho$, где $\varrho=1+a \xi_{0}$. Этой замене соответствует соотношение $D_{0}=\frac{1}{\varrho} D_{x}$. Получим

$$
\left.\left\{D_{x}^{4}-a^{2} D_{x}^{2}-l_{1}^{4} \mid \alpha(c-d \varrho) \varrho-\frac{\beta^{2}}{\varrho^{2}}\right]\right\} w=0
$$

Разложим $\mathrm{Q}=e^{a x}$ в ряд

$$
\begin{aligned}
e^{a x} & =1+a x+\frac{a^{2} x^{2}}{2}+\ldots, \\
e^{-2 a x} & =1-2 a x+2 a^{2} x^{2}+\ldots, \\
\alpha & =\alpha_{0}+a \alpha_{1}+a^{2} \alpha_{2}+\ldots .
\end{aligned}
$$

Уравнение (5) принимает вид

$$
\begin{aligned}
& D_{x}^{4} w-a^{2} D_{x}^{2} w-l_{1}^{4}\left\{( \alpha _ { 0 } + a \alpha _ { 1 } + a ^ { 2 } \alpha _ { 2 } ) \left[c\left(1+a x+\frac{a^{2} x^{2}}{2}\right)-\right.\right. \\
& \left.\left.-d\left(1+2 a x+2 a^{2} x^{2}\right)\right]-\beta^{2}\left(1-2 a x+2 a^{2} x^{2}\right)\right\} w=0 .
\end{aligned}
$$

Рассмотрим случай защемленных краев, т. е. $w=0, D_{x} w=0$ на обонх краях.

\section{Установим пределы изменения $x$ :}

$$
\ln \left(1+a \xi_{0}\right)=a \xi_{0}-\frac{a^{2} \xi_{0}^{2}}{2}+\frac{a^{3} \xi_{0}^{3}}{3}-\cdots, x=\xi_{0}-\frac{a}{2} \xi_{0}^{2}+\frac{a^{2}}{3} \xi_{0}^{3}-\cdots
$$

Если $\xi_{0}=\xi^{\prime}$, то $x=-1+\frac{a^{2}}{6}$, если $\xi_{0}=\xi^{\prime \prime}$, то $x=1-\frac{a^{2}}{6}$. Учитывая, что $a$ - малый параметр, возьмем за промежуток нзменения $-1 \leqslant x \leqslant 1$. 
В уравнении (7) примем $a$ за параметр возмущения. Параметр $\beta^{2}$ сохраняет свое значение во всех приближениях. Собственные значения $\alpha_{0}, \alpha_{1}, \alpha_{2}, \ldots$ находим последовательно по обычным правилам метода

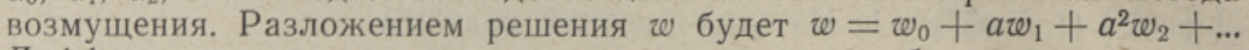
Дифференциальное уравнение для нулевого приближения получим из (7) в виде

$$
\left\{D_{x}^{4}-l_{1}^{4}\left[\alpha_{0}(c-d)-\beta^{2}\right]\right\} w_{0}=0
$$

Обозначим $u^{4}=\alpha_{0}(c-d)-\beta^{2}$. Уравнению (8) удовлетворяет симметричная функция

$$
w_{0}=A \cos u l_{1} x+B \operatorname{ch} u l_{1} x
$$

Чтобы краевые условия на краю $x=1$ удовлетворялись, величина $u l_{1}=\frac{x}{2}$ должна удовлетворять уравнению $\operatorname{tg} u l_{1}+$ th $u l_{1}=0$. Наименьшее собственное значение соответствует величине $u l_{1}=2,365$, или $x_{0}=4,730$. Из определений $\alpha_{0}$ и $\beta^{2}$ получим для нулевого приближения нагрузки $q_{10}$ выражение

$$
q_{10}=\frac{a_{0} \lambda \cos ^{2} \varphi}{\beta}
$$

Отсюда вычислим нулевое приближение минимальной критической нагрузки

$$
q_{10 \min }=\frac{4}{3} \sqrt{3}^{3} \frac{1}{c-d} \frac{x_{0}}{l}(\lambda \cos \varphi)^{3 / 2}
$$

которому соответствует

$$
s^{2}=\frac{x_{0}}{l} \sqrt[4]{\frac{3 \cos ^{2} \varphi}{\lambda^{2}}}, \quad B=0,1329 A .
$$

Для нахождения первой поправки функции прогиба $ш$ обозначим $u l_{1} x=x_{1}$, тогда $D_{x}=u l_{1} D_{x_{1}}$ и на основании $(7)$ получим для $w_{1}$ уравнение

$$
\left(D_{x_{1}}^{4}-1\right) w_{1}=\frac{1}{u^{4}}\left\{(c-d) \alpha_{1}+\left[(c-2 d) \alpha_{0}+2 \beta^{2}\right] \frac{x_{1}}{u l_{1}}\right\} w_{0} .
$$

В этом уравнении собственное значение $\alpha_{1}=0$, так как ему соответствует нечетная собственная функция. При этом достаточно удовлетворение краевых условий только на одном краю.

Из определения $\beta^{2}, u^{4}$ и из выражения (12) следует, что $\frac{\beta^{2}}{u^{4}}=3$; кроме того, поскольку $\alpha_{0}=\frac{u^{4}+\beta^{2}}{c-d}$, то можем вместо (13) написать

$$
\left(D_{x_{1}}^{4}-1\right) w_{1}=\left(4 \frac{c-2 d}{c-d}+6\right) \frac{x_{1}}{u l_{1}} w_{0} .
$$

- Обозначим $b=4 \frac{c-2 d}{c-d}+6$.

После интегрирования и определения постоянных получим

$$
w_{1}=\frac{1}{8} \frac{b}{u l_{1}}\left[x_{1}^{2}-\left(u l_{1}\right)^{2}\right] D_{x_{1}} w_{0}-\frac{3}{8} \frac{b}{u l_{1}} x_{1} w_{0} .
$$


Аналогично получим вторую поправку $w_{2}$ и собственное значение

$$
\alpha_{2}=\left(-0,054 \frac{(c-2 d)^{2}}{(c-d)^{2}}+\frac{-0,20 c+0,50 d}{c-d}+0,035\right) \alpha_{0}
$$

и наша искомая минимальная критическая нагрузка в размерном виде

$$
\begin{gathered}
p_{1}=\frac{V \overline{6}}{9(c-d)} \frac{x_{0} E h^{3 / 2}}{l\left(1-v^{2}\right)^{3 / 4}}\left(\frac{\cos \varphi}{\varrho_{0}}\right)^{3 / 2}\left[1+a^{2}\left(-0,054 \frac{(c-2 d)^{2}}{(c-d)^{2}}+\right.\right. \\
\left.\left.+\frac{-0,20 c+0,50 d}{c-d}+0,035\right)\right]
\end{gathered}
$$

причем $c=\frac{\varrho_{2}}{\varrho_{2}-\varrho_{1}}+\frac{p_{2}}{p_{1}}, \quad d=\frac{\varrho_{0}}{\varrho_{2}-\varrho_{1}}$.

Рассмотрим некоторые частные случаи. При $p_{2}=0, c-d=$ $=\frac{\varrho_{2}-\varrho_{0}}{\varrho_{2}-\varrho_{1}} \approx \frac{1}{2}, d=\frac{1}{2 a}$ и выражение в квадратных скобках (17) принимает вид

$$
1+a^{2}\left(-0,216 d^{2}+0,81 d-0,22\right)
$$

или

$$
1-0,054+0,40 a-0,22 a^{2} .
$$

При $p_{1}=0 . p_{2} \neq 0$ это выражение совпадает с результатом, полученным в работе $\left.{ }^{1}\right]$ :

$$
1-0,22 a^{2} .
$$

Нулевому приближению критической нагрузки $p_{10} \min$ соответствует

$$
s_{\text {min }}^{2}=\frac{\chi_{0}}{L} \sqrt{\frac{6 Q_{0}^{3} \cos \varphi}{h}} \sqrt[1]{1-\nu^{2}} .
$$

\begin{tabular}{|c|c|c|c|c|c|c|c|c|}
\hline \multirow[t]{2}{*}{ 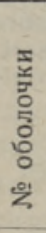 } & \multirow[t]{2}{*}{$\begin{array}{c}\text { Материал } \\
\text { оболочки }\end{array}$} & $\begin{array}{c}\text { Длина по } \\
\text { образую- } \\
\text { щей }\end{array}$ & $\begin{array}{c}\text { Средний } \\
\text { радиус }\end{array}$ & Толщина & \multirow{2}{*}{$\begin{array}{r}2 \\
1 \\
0 \\
8 \\
511 \\
5 \\
5 \\
5\end{array}$} & \multirow[t]{2}{*}{$\begin{array}{c}\text { Число } \\
\text { волн по } \\
\text { окруж- } \\
\text { ности }\end{array}$} & 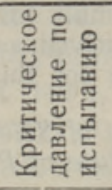 & $\begin{array}{c}\text { Расчетное } \\
\text { мини- } \\
\text { мальное } \\
\text { давление } \\
p_{2}\end{array}$ \\
\hline & & & MM & & & & \multicolumn{2}{|c|}{$\mathrm{Kr} / \mathrm{CM}^{2}$} \\
\hline $\begin{array}{l}1 \\
2 \\
3 \\
4 \\
5\end{array}$ & $\left|\begin{array}{l}\text { Сталь } \\
\text { Сталь } \\
\text { Дюраль } \\
\text { Сталь } 40 \mathrm{X} \\
\text { Сталь } 45\end{array}\right|$ & $\begin{array}{l}176 \\
140 \\
140 \\
203 \\
203\end{array}$ & $\begin{array}{l}28,65 \\
28,58 \\
28,58 \\
27,50 \\
35,43\end{array}$ & $\begin{array}{l}0,3 \\
0,3 \\
0,3 \\
0,6 \\
0,6\end{array}$ & $\begin{array}{l}86 \\
85 \\
85 \\
81 \\
85\end{array}$ & $\begin{array}{l}4 \\
4 \\
4 \\
3 \\
4\end{array}$ & $\begin{array}{l}5,33 \\
5,33 \\
2,5 \\
21,33 \\
17\end{array}$ & $\begin{array}{r}4,70 \\
4,72 \\
1,68 \\
23,67 \\
19,47\end{array}$ \\
\hline
\end{tabular}

Подставляя из формулы (20) выражение для $\frac{\varkappa_{0}}{L}$ в (17), получим для нулевого приближения критической нагрузки

$$
p_{10 \mathrm{~m} \ln }=\frac{F s^{2}{ }_{\min } \cos \varphi}{9(c-d)\left(1-v^{2}\right)}\left(\frac{h}{\varrho_{0}}\right)^{3}
$$

таблица 1

Если в формулу (21) подставить $c-d=1$, то получим выражение для определения минимальной критической нагрузки при равномерной нагрузке, которое обозначалось через $p_{2}$. Сравнение с экспериментальными данными $\left[{ }^{2}\right]$ приведено в табл. 1 . 


\section{Л ИТЕРА Т У Р А}

1, П. В. Мюр се пп, Об устойчивости кругового усеченного конуса под действием равномерно распределенного внешнего давления, Известия АН ЭССР. Серия техн, и физ.-мат. наук, т. VII, № 2, 1958.

2. И. И. Тр а пезин, Об устойчивости конической оболочки, находящейся под гидростатическим давлением, Сб. Расчеты на прочность, жесткость, устойчивость и колебания, Машгиз, М. 1955.

Институт физики и астрономии

Академии Наук Эстонской ССР

Поступила в редакцию

18. X 1960

\section{KOONILISE POOORDKOORIKU KRIITILISE KOORMISE MÄARAMISEST}

\section{P. Müürsepp}

\section{Resümee}

Häiremeetodi abil tuletatakse lihtne arvutusvalem keskmise pikkusega tüvikoonusekujulise pöördkooriku kriitilise koormise määramiseks, sel juhul kui koorik on piki koonuse moodustajat lineaarselt jaotatud välisrōhu all (joon. 1).

Erijuhuna saadakse valem kriitilise koormise määramiseks ühtlase välisrōhu puhul. Selle juhu jaoks esitatakse tabelis 1 teoreetiliste tulemuste võrdlus katseandmetega.

Füüsika ja Astronoomia Instituut

Eesti NSV Teaduste Akadeemia
Saabus toimetusse

18. X 1960

\section{ON THE DETERMINATION OF THE CRITICAL LOAD OF CIRCULAR CONE-SHAPED SHELLS}

\section{P. Müürsepp}

Summary

A simple formula for the determination of the critical load of circular frustums of cone-shaped shells of medium length has been derived by means of the perturbation method, when the shell is under external pressure distributed linearly along the generatrix of the cone (Fig. 1).

The formula for the determination of the critical load under uniform external pressure has been derived as a special case. Theoretical results have been compared with experimental data for this case (Table 1).

Academy of Sciences of the Estonian S.S.R., Institute of Physics and Astronomy

Received

Oct. 18th. 1960 